\title{
A survey of paediatricians' practice and training in routine infant eye examination
}

\author{
Jugnoo S Rahi, Richard Lynn
}

\begin{abstract}
A survey of a sample of UK paediatricians was carried out to identify the practices and determine the training of those involved in routine surveillance examinations to detect ophthalmic disorders in infants. The findings indicate important variation in current practices and raise concerns about both undergraduate and postgraduate training in ophthalmic assessment of infants.
\end{abstract}

(Arch Dis Child 1998;78:364-366)

Keywords: screening; surveillance; vision

Early detection of treatable sight or life threatening ophthalmic disorders in infants is essential for their optimal management. Consequently, routine examinations to identify them, during the neonatal period and again at 6-8 weeks of age, are an established component of child health surveillance in Britain. Recommendations about practice are made in Health for All Children ${ }^{1}$ and Ophthalmic Services for Children, ${ }^{2}$ the reports of two national joint working parties. Careful inspection, evaluation of the red reflex, examination for the presence of squint, and assessment of visual behaviour are advised for the detection of serious sight threatening disorders, notably congenital eye anomalies such as microphthalmos and coloboma, congenital cataract, glaucoma, and retinoblastoma. However, little is known about the extent to which these recommendations have been adopted and the training of those involved.

Accepted 10 November 1997

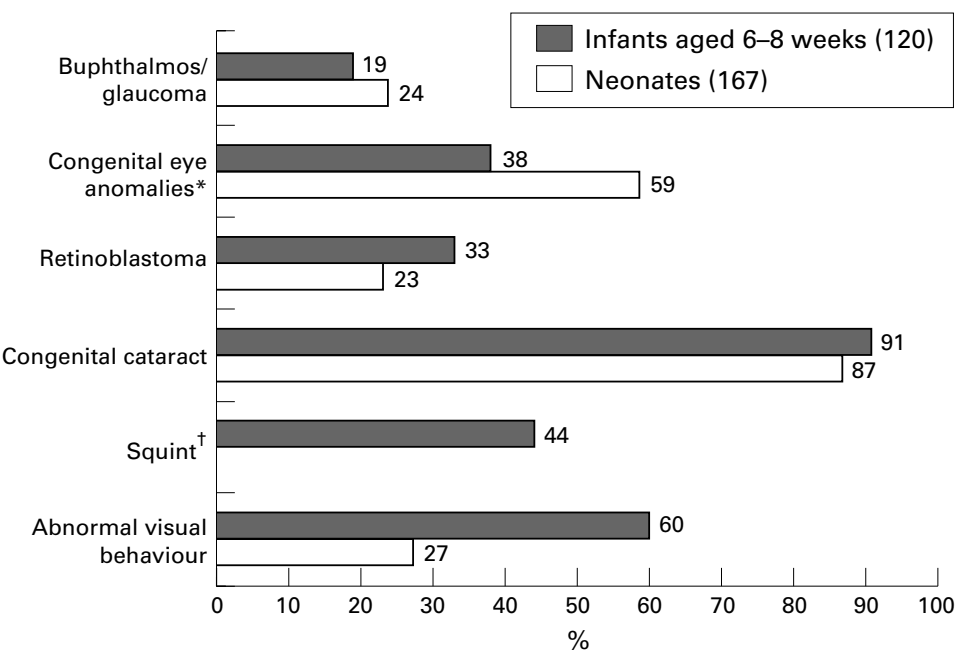

Figure 1 Percentage of consultants seeking specific abnormalities in neonates and infants aged 6-8 weeks. ${ }^{\star}$ Includes all congenital eye anomalies, for example microphthalmos and coloboma; tnot applicable in neonates.

\begin{abstract}
Methods
In 1995, a survey of a representative sample of UK paediatricians was carried out to identify the practices and describe the training of those currently responsible for the routine examinations of young infants. After a pilot study, an anonymised semistructured questionnaire was sent to hospital and community consultants (250), hospital based trainees (150), and clinical medical officers (CMOs) (100) selected randomly from membership lists of the Royal College of Paediatrics and Child Health, the British Association of Perinatal Medicine, the British Association of Community Child Health, and the Society for Public Health. Consultant paediatricians were surveyed because, even when not personally involved in routine examinations of infants, they would be responsible for the provision of the service, including the training of junior colleagues. Respondents were asked to list the ophthalmic abnormalities and disorders they specifically sought in infants in each age group applicable to their practice and to report any previous training in ophthalmic examination of infants and their requirements for further training.
\end{abstract}

\section{Results}

After one reminder, 365 (73\%) paediatricians returned completed questionnaires (205 consultants, 102 hospital paediatric trainees, and $58 \mathrm{CMOs})$. Of these respondents, 272 indicated that they were responsible for examinations of neonates and 200 for examinations of infants aged 6-8 weeks: the percentage of these respondents reporting seeking the ophthalmic disorders specified in the national recommendations ${ }^{12}$ for infants aged up to 8 weeks is shown in fig 1 (consultants) and fig 2 (hospital juniors and CMOs). There were no substantial or consistent differences between the these two groups or between community versus hospital based paediatricians. Congenital cataract was the most frequently sought disorder at both examinations by all groups.

A fifth of all respondents (75) and a third (32) of hospital paediatric trainees reported receiving no training in the ophthalmological examination of infants. Of those reporting some training, $57 \%$ had received this only as postgraduates and $16 \%$ only while undergraduates. Respondents had been trained in ophthalmic examination by a senior paediatrician $(80 \%)$, an ophthalmologist $(41 \%)$, an orthoptist $(18 \%)$, or a paediatric trainee colleague $(4 \%)$ with a quarter receiving training from more than one source. The majority $(92 \%)$ of these respondents had received some practical training with $46 \%$ reporting receiving 


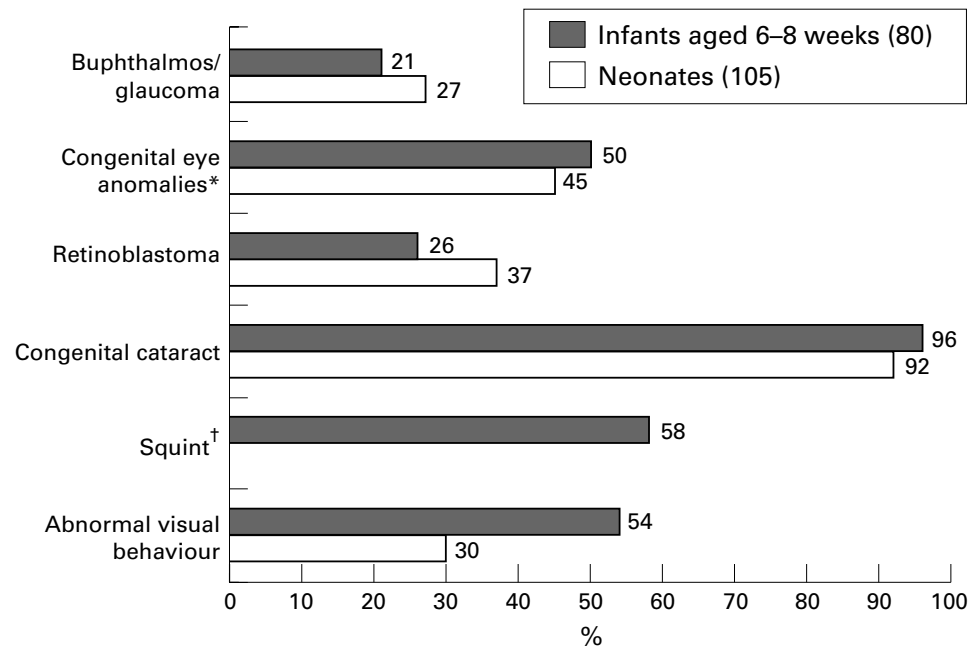

Figure 2 Percentage of hospital based paediatric trainees and CMOs seeking specific abnormalities in neonates and infants aged 6-8 weeks. ${ }^{\star}$ Includes all congenital eye anomalies, for example microphthalmos and coloboma; tnot applicable in neonates.

some formal teaching in addition. Overall $71 \%$ (248) of all responding paediatricians $(57 \%$ of consultants, $81 \%$ of hospital trainees and CMOs) considered they would benefit from further training by an ophthalmologist. Most sought practical training only $(49 \%)$ or combined with formal teaching $(40 \%)$.

\section{Discussion}

Serious sight or life threatening ocular disorders, such as congenital cataract, congenital eye anomalies, retinoblastoma and glaucoma, are uncommon, with a combined prevalence of the order of 0.5 per 1000 births. ${ }^{1}$ The main purpose of routinely examining young infants to detect such disorders is to ensure that effective treatment and relevant medical and educational advice and support for the family of the affected child, are provided at the earliest opportunity. ${ }^{12}$ The effectiveness of these examinations is assumed in ophthalmic surveillance or vision screening programmes for older children, which are primarily intended to confirm normal visual development and to detect disorders such as squint or amblyopia which may develop during childhood. ${ }^{12}$

The survey findings indicate important variation in the implementation of current recommendations about screening and surveillance for ophthalmic disorders in infancy. The degree of clinical experience of respondents and the setting in which they work do not appear to be the main factors influencing the variation in practice, as this did not differ substantially between consultants and trainees or hospital and community based paediatricians. Ophthalmic assessment of infants is difficult and requires specific knowledge and practical training to do effectively. At the time of this survey routine neonatal examinations were carried out by junior hospital based trainee paediatricians but recently, in some areas, this has become the responsibility of general practitioners or midwives, who were not surveyed. There is increasing interest in standardising the content of the routine neonatal examination and in identifying the most appropriate health care professionals to undertake $i^{3}$ : this presents a good opportunity to review the ophthalmic component and to develop appropriate training programmes for those undertaking it.

The survey findings raise serious concerns about the extent and content of both undergraduate and postgraduate medical training in ophthalmic examination of infants, especially the low reported frequency of training received from ophthalmologists and other ophthalmic professionals. Perhaps most important is the finding that most paediatricians, even consultants, considered they would benefit from further training. This is consistent with the reported training needs of general practitioners involved in child health surveillance, ${ }^{4}$ who are increasingly responsible for the routine examination at 6-8 weeks of age, ${ }^{5}$ some of whom may not have received appropriate undergraduate training in ophthalmic assessment. Variation in current postgraduate training provision for general practitioners and others involved in child health surveillance has already been identified. ${ }^{5}$ Undergraduate ophthalmology training, traditionally during a separate clinical attachment, might be improved by greater integration with the main medical specialties: for example, undergraduates could attend paediatric ophthalmology clinics during their attachment to the paediatric department. Increased integration would enhance understanding of the relevance of ophthalmology to other disciplines, improve knowledge about specific ophthalmic disorders and provide greater opportunities to practise examination techniques. Postgraduate training for all doctors involved in screening and surveillance examinations should include specific teaching on paediatric ophthalmic disorders and visual assessment of children together with an evaluation of the skills acquired. The interdisciplinary discussions necessary for the development of such programmes for paediatricians have already been advocated. ${ }^{2}$ This is an opportunity to explore the possible role of orthoptists as trainers, given their expertise in the assessment of vision and ocular motility in children. A minority of respondents reported receiving any training from an orthoptist despite the scope which exists in both community and hospital settings, especially where secondary screening of referred children is undertaken by orthoptists. While development of undergraduate and postgraduate training programmes is a long term process, closer liaison between paediatric and ophthalmology departments could create training opportunities in the short term: for example, joint assessment clinics for children with visual impairment, already advocated to improve overall management, ${ }^{2}$ offer excellent scope for further training of paediatricians and ophthalmologists alike.

We suggest that optimal ophthalmic surveillance of newborn and young infants nationally requires more specific guidance on the purpose and content of examinations as well as the programmes for training and assessment of all those involved, both now and in the future. In order to strengthen and develop such 
programmes it will be necessary to gather the research evidence to inform practice which, as in some other areas of infant screening, ${ }^{6}$ is currently lacking: there is significant scope for this at a time when other aspects of child surveillance are being re-evaluated.

Conflict of interest : None

We thank all the paediatricians who took part in the survey, for completing the questionnaire and for providing helpful suggesHealth) and Dr Gardner (Society for Public Health) for help with distribution of questionnaires; Dr Carol Dezateux and with distribution of questionnaires; Dr Carol Dezateux and
Professor Catherine Peckham (Institute of Child Health, Professor Catherine Peckham
London) for their comments.

Jugnoo Rahi is supported by the Medical Research Council.
1 Hall DMB, ed. Health for all children. 3rd Ed. Report of the Third Joint Working Party on Child Health Surveillance. Oxford: Oxford University Press, 1996.

2 Royal College of Ophthalmologists and the British Paediatric Association, UK. Ophthalmic services for children. Report of a Joint Working Party. London: Royal College of Ophthalmologists and the British Paediatric Association, 1994.

3 Court S. Examination of the newborn-for what and by whom? Changing Childbirth Update September 1995;3:3 (paper).

4 Glickman M, Gillam S, Boyle G, Woodroffe C. What makes general practitioners do child health surveillance? Arch Dis Child 1994;70:47-50.

5 Butler J, Freidenfeld K, Relton J. Child health surveillance in the new NHS. Summary report commissioned by the Department of Health. Canterbury: Centre for Health Services Studies, University of Kent, August 1995.

6 Dezateux C, Godward S. A national survey of screening for congenital dislocation of the hip. Arch Dis Child 1996;74: $445-8$ 